TRANSFER

Reception Studies

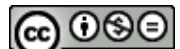

\title{
„Können Tränen meiner Wangen nichts erlangen?“ Manifestationen der Traurigkeit in der deutschen Musik des 18. Jahrhunderts
}

\section{"Will my tears change anything?" Manifestations of sadness in German music of the 18 th century}

\begin{abstract}
Emotions and feelings, such as sadness, disappointment or doubt, are not only a characteristic feature of the narrative present in the widely understood German-language literature. Manifestations of various emotional states can undoubtedly be found in numerous vocal and instrumental works, whose period of splendor appears during the 18th century, in particular in the decades within the time frame set by the St. John Passion of Johann Sebastian Bach BWV 245 (1724) and the majestic oratorios The Creation and The Seven Last Words of Our Saviour on the Cross by Joseph Haydn (1796-98). The purpose of this article is to show the affective potential of selected texts in the orbit of the impact of the phenomenon of musical rhetoric and to illuminate the works of famous German composers of the 18th century, placed in a broader cultural and literary context.
\end{abstract}

Keywords: Johann Sebastian Bach's passion music, Viennese Classics, emotions and affects.

In der abendländischen Geistesgeschichte gibt es zahlreiche Zeugnisse darüber, dass traumatische Erlebnisse, insbesondere die Todeserfahrung, bei den betroffenen Menschen nicht nur unmittelbare Spuren in der philosophischen Besinnung hinterlassen, sondern gleichzeitig imstande sind, sowohl in individueller als auch kollektiver Hinsicht zur religiösen Bekehrung beizutragen. Umgekehrt kann der Verlust haltgebender Daseinsformen eine 
geistige und existenzielle Krise verursachen, denn „in der Erfahrung des Todes anderer und der daraus resultierenden Trauer wird der Mensch meist in unvermittelter Härte mit der unheimlichen Rätselhaftigkeit und Unkalkulierbarkeit des Todes konfrontiert."1

Affektvolle Manifestationen der Trauer begleiteten von Anfang an nicht nur die Entwicklung der literarischen Gattungen, sondern sie manifestieren sich ebenso in unterschiedlichen musikalischen Formen. ${ }^{2}$ Selbst in den früh entstandenen Liedern, die anlässlich von Bestattungen in der Form des Threnos bzw. des Epikedeion aufgeführt wurden, wurde zu Lebzeiten die Klage über den Tod von Helden und das Lob ihrer Taten transportiert. Darüber hinaus wurden die Lieder infolge ihrer gattungsbezogenen Entwicklung nicht nur zur Manifestation von legendenumwobenen Inhalte und Mythen, sondern sie erfüllten zunehmend eine pragmatische Funktion, nämlich die Hinterbliebenen in ihrer Trauer zu trösten. ${ }^{3}$

\section{Trauermanifestationen in der Passionsmusik von Johann Sebastian Bach}

Aus der heutigen Perspektive kann man die These wagen (worauf Kurt von Fischer aufmerksam macht), dass die begriffliche Verbindung zwischen der Passionsmusik und Johann Sebastian Bach im Laufe der Zeit so eng geworden ist, dass die Gefahr besteht, die Passionen des Thomaskantors losgelöst von ihrem geschichtlichen und liturgischen Rahmen zu sehen und zu hören. Hierbei wird häufig nicht berücksichtigt, dass die Passion als Gattung in einer jahrhundertealten musikalischen und frömmigkeitsgeschichtlichen Tradition steht: „Schon in den ersten christlichen Jahrhunderten“, so Fischer, "gehörten Passionslesungen zu den zentralen Bestandteilen der Karwochenliturgie. Im Laufe der Zeiten hat sich, nicht zuletzt unter dem Einfluß eines

1 Ulrike Schaeben, Trauer im humanistischen Dialog (München/Leipzig: Saur Verlag, 2002), 1.

2 Dabei bleibt die Frage relevant, nach welchen Kriterien die Emotionen in der Musik und ihre Wirkung auf den Rezipienten eingestuft werden können. Ansatzweise erklärt Willy Pastor die Grundlagen dieses Phänomens: „Auf zwei Tongeschlechtern bauen all unsere Lieder und Weisen sich auf: auf dem Dur und dem Moll. Wie jedes Kind weiß, klingt das Dur für uns freudig, das Moll dagegen traurig. Dur- und Mollgeschlecht aber sind verschieden nur in zwei bis drei Intervallen, der Terz, Sext und Septime: bei dem Dur erscheinen diese Intervalle in der großen, bei Moll in der kleinen Form. "Willy Pastor, Die Geburt der Musik. Eine Kulturstudie (Leipzig: Fritz Eckardt Verlag, 1910), 110-111.

3 Vgl. Hans-Herwarth. Studnik, Die consolatio mortis in Senecas Briefen, zit. nach: Schaeben, Trauer im humanistischen Dialog, 6. 
sich wandelnden Verständnisses von Jesu Leidensgeschichte, die Passion allmählich zu einer eigenen Gattung herausgebildet."4

Die Hauptlinien der Entwicklung der Passionsmusik sind naturgemäß nicht von ihrem sozialen und kulturellen Hintergrund zu trennen und zwar angesichts des Umstands, dass die Passionsaufführungen von Anfang an in engem Zusammenhang mit der Entstehung der religiösen und konfessionellen Strömungen standen. Auf deutschem Boden ist vor diesem Hintergrund vor allem der Einfluss des Pietismus von Bedeutung; die darin präsente Beschäftigung mit dem eigenen Innen- und Seelenleben, und damit auch das Bedürfnis nach mitleidender Teilnahme an der Passion, „ist für den Pietisten von großer Bedeutung: Der Sinn für religiöse Stimmungen und persönliche Frömmigkeitsgefühle wird aktiviert. Dies hat sich auch auf die Passionsmusiken bis hin zu Bach ausgewirkt."5

Die erste der beiden großen Passionen von Johann Sebastian Bach, die überliefert sind, also die Johannes-Passion, wurde in ihrer ursprünglichen Fassung mit größter Wahrscheinlichkeit in der Karwoche 1724 im Gottesdienst uraufgeführt. Auffällig ist die Zweiteiligkeit des Werkes, die aber nicht als besondere Erfindung des Thomaskantors eingestuft werden kann, da sie sich auch in anderen oratorischen Passionen in der Zeit um 1700 findet. Die formale Konstruktion der Passion kann man vor allem auf den Umstand zurückführen, dass zwischen beide Teile gewöhnlich noch die Predigt eingeschoben wurde. ${ }^{6}$

Auch wenn die Bedeutung der Johannes-Passion für die Musikgeschichte außer Frage steht - man kann sie folglich als ein buntes Mosaik unterschied-

4 Kurt von Fischer, „Historische und frömmigkeitsgeschichtliche Traditionen der Passion“, in Johann Sebastian Bach, Johannes-Passion [The Monteverdi Choir, The English Baroque Soloists, John Eliot Gardiner], Hamburg: Polydor International, 1986, 10. Fischer betont auch die Entwicklung der Passionsmusik von J. S. Bach vor dem Hintergrund der zunehmenden Popularisierung der neuen Gattung, die gewöhnlich als „oratorische Passion“ bezeichnet wird: „Die Geschichte derjenigen musikalischen Passion, die ihren unerreichten Höhepunkt in den Werken Johann Sebastian Bachs finden sollte, beginnt recht eigentlich in der zweiten Hälfte des 17. Jahrhunderts. Es handelt sich dabei um einen Typus, den man zu Recht als oratorische Passion bezeichnet hat. Seine wesentlichsten Elemente sind: 1. neu komponierte Rezitative, die sich durch ihre Expressivität und Sprachbezogenheit im Laufe der Zeit, vor allem seit dem Beginn des 18. Jahrhunderts, dem Opernrezitativ angenähert haben. 2. Einfügung von Gemeinde-Chorälen (dies in der Praxis schon seit dem ausgehenden 16. Jahrhundert); 3. Einfügung von nicht dem Passionstext entnommenen oder neu gedichteten lyrischen Texten und 4. Mitbeteiligung von Instrumenten und allmählich immer stärkerer Ausbau der instrumentalen Elemente. "Von Fischer, Historische und frömmigkeitsgeschichtliche Traditionen der Passion, 11.

5 von Fischer, Historische und frömmigkeitsgeschichtliche Traditionen der Passion, 11.

6 Vgl. von Fischer, Historische und frömmigkeitsgeschichtliche Traditionen der Passion, 13. 
licher musikalischer Kleinformen analysieren und dabei verschiedene Rezeptionsaspekte hervorheben -, bleiben für das Werk vor allem das emotionale Potenzial und die meisterhafte Darstellung zahlreicher Affekte und Stimmungen signifikant. Obwohl also die Johannes-Passion als eine stimmungsvolle, affektgeladene und facettenreiche Manifestation der Trauer und Traurigkeit eingestuft werden kann, erscheint in ihr dennoch ein explizites Motiv des Weinens bzw. der Tränen nur an einer Stelle, die wortwörtlich dem Bibeltext entnommen wurde: „Da verleugnete Petrus abermal, und alsobald krähete der Hahn. Da gedachte Petrus an die Worte Jesu und ging hinaus und weinete bitterlich." 7 Unabhängig davon wirkt, im Gegensatz zur Matthäus-Passion - worauf nochmals Fischer verweist - „mit ihren zahlreichen lyrischen Arien und Ariosi und ihrem in sich geschlossenen tonartlichen Plan [...] die Johannes-Passion mit ihren Gerichtsszenen dramatischer und in mancher Hinsicht auch kühner."8

Was die Bedeutung beider Passionen von Bach für die Musikgeschichte und ihren künstlerischen Rang anbelangt, erscheint - obwohl die JohannesPassion von einer einzigartigen, melancholischen und trauervollen Stimmung durchdrungen ist - erst das später entstandene Werk als Beispiel für emotionsgeladene Kunst der Superlative. Nicht umsonst pflegte man schon im Bachschen Familienkreis - und bis heute ist diese Tendenz in musikwissenschaftlich fundierten Kategorisierungen erhalten geblieben - eben die Matthäus-Passion als „die große Passion“ zu bezeichnen. Und gerade dieses Prädikat ist auch fest in einer langen Tradition verankert: Wenn nämlich die Gattin des Komponisten, Anna Magdalena Bach, auf eine Generalbassstimme den der Rechtschreibung nicht ganz entsprechenden Vermerk „zur groß Bassion" schrieb, so wusste man auch im Hause des Thomaskantors sehr genau, wohin diese Stimme gehörte. ${ }^{9}$

Zur Entstehung dieses in jeder Hinsicht prächtigen und durchgeistigten Werks ${ }^{10}$ lässt sich sagen, dass die 1729 uraufgeführte Passion zeitlich zwi-

7 Johann Sebastian Bach, Johannes-Passion [The Monteverdi Choir, The English Baroque Soloists, John Eliot Gardiner] (Hamburg: Polydor International, 1986), 40. Ein ähnliches Motiv erscheint in einer ausdrucksstarken Form ebenfalls in der Matthäus-Passion, ausführlich beschreibt es Elizabeth Joyner im On-Line-Artikel. Vgl. Elizabeth Joyner, Erbarme Dich. The Temptation of Peter in Bach's St. Matthew Passion, https://earthandaltarmag.com/ posts/erbarmedich-the-temptation-of-peter-in-bachs-st-matthew-passion.

8 von Fischer, Historische und frömmigkeitsgeschichtliche Traditionen der Passion, 13.

9 Vgl. Christoph Wolff, „Bachs ,Grosse Passion’“, in Johann Sebastian Bach, Matthäus-Passion [The Monteverdi Choir, The London Oratory Junior Choir, The English Baroque Soloists, John Eliot Gardiner] (Hamburg Deutsche Grammophon, 1989), 12.

10 Auffällig ist der nahezu transzendente Charakter der Matthäus-Passion. In Anlehnung an Fritz Jöde kann man in diesem Zusammenhang auf die rückwirkenden Relationen zwi- 
schen die in bescheidenerem Format gehaltenen Passionen nach Johannes von 1724 und nach Markus von 1731 gehört. Was sie jedoch von den beiden soeben genannten musikalischen Meisterwerken unterscheidet, ist die Tatsache, dass, obwohl Bach bekanntlich bei Wiederaufführungen - vor allem von größeren Werken - nahezu immer Anlass zu Änderungen und Verbesserungen fand, sich diese im Falle der Matthäus-Passion lediglich auf eine ganz bestimmte Tendenz beschränkten, nämlich auf die „weitere Monumentalisierung durch Erweiterung der musikalischen Struktur und des Aufführungsapparates."11 Darüber hinaus birgt die Matthäus-Passion ein auffällig großes emotionales Potenzial in sich, insbesondere hinsichtlich der Darstellungen von Trauer, die in zahlreichen musikalischen Ausdrucksformen zur Geltung kommen. Es ist also nicht verwunderlich, dass in der vor nahezu einhundert Jahren erschienenen großformatigen Publikation Geschichte der deutschen Musik von Hans Joachim Moser auf den formalen, inhaltlichen und affektiven Reichtum der "großen Passion“ aufmerksam gemacht wird:

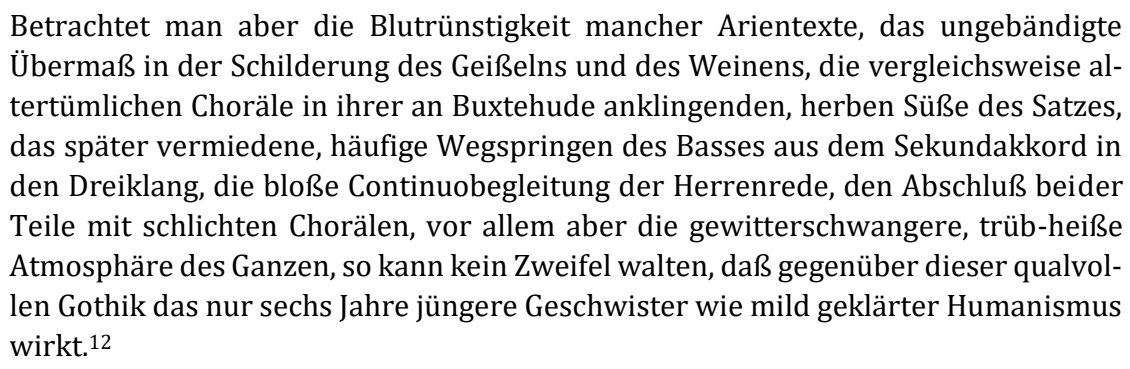

Besonders signifikant für das in den Jahren 1727-29 entstandene Werk sind allerdings die Manifestationen der Traurigkeit, die sich unmittelbar aus der facettenreich und ausdrucksstark dargestellten Leidensgeschichte Jesu ergeben. Auf eine bildhafte Weise wird diese in der "großen Passion“ prä-

schen der Musik und Gesinnung verweisen: „Musik ist für uns keine Stimmungsangelegenheit mehr, erfüllt durch mechanisch-technische Fertigkeiten, sondern eine Gesinnungsangelegenheit. Wir glauben, daß sie, wo sie der Mensch einmal aus sich herausgestellt hat, nach eigenen, über ihm stehenden Gesetzen dahinschreitet." F. Jöde, Musik und Erziehung. Ein pädagogischer Versuch und eine Reihe Lebensbilder aus der Schule (Berlin: Georg Kallmeyer Verlag, 1932), 19.

11 Wolff, Bachs „Grosse Passion", 12. Hierzu ist noch zu ergänzen, dass bei der höchstwahrscheinlich dritten Aufführung des Werkes im Jahre 1736 der ursprünglich schlichte Schlusschoral des Ersten Teils (,Jesum lass ich nicht von mir") durch den aus der Zweitfassung der Johannes-Passion übernommenen, großangelegten Choralchorsatz „O Mensch, bewein dein Sünde groß" ersetzt wurde. Vgl. Wolff, Bachs „Grosse Passion“.

12 Hans Joachim Moser, Geschichte der deutschen Musik. Vom Beginn des dreißigjährigen Krieges bis zum Tode Joseph Haydns (Stuttgart und Berlin: J. S. Cotta'sche Buchhandlung Nachfolger, 1923), 222. 
sente Tendenz in der Interpretation des schon oben zitierten Musikwissenschaftlers Moser beschrieben. ${ }^{13}$ Des Weiteren ist für das Verständnis von Bachs künstlerischem Vorgehen in der musikalischen Disposition der Passionsgeschichte der Umstand von Bedeutung, dass hier vor allem die Art der planvollen und beziehungsreichen Aneinanderreihung und der absolut unschematischen Verwendung der musikalischen Formen und Kompositionsarten im Dienst dramatischer Gestaltung erscheint. ${ }^{14}$

Eine solche Tendenz lässt sich schon im Eingangschor „Kommt, ihr Töchter, helft mir klagen" ausmachen, denn ausgerechnet in dieser allegorisierenden Klagedichtung Picanders - worauf Christoph Wolff verweist - „vertont Bach im Stil des französischen Tombeau, speziell als Trauermarsch der Schar der Gläubigen, die den Berg Zion zur heiligen Stadt Jerusalem hinaufzieht. 'Die Tochter Zion' ist personifizierte Stätte des Leidens, der Passion Jesu.“15

Nicht weniger ausdruckstark ist im Eingangschor die polichoral gestellte Frage, deren Emotionalität bereits auf der Textebene deutlich zum Vorschein kommt: „Kommt ihr Töchter, helft mir klagen / Sehet - Wen? - den Bräutigam. / Seht ihn - Wie? - als wie ein Lamm!"16 Eine so konzipierte Anwendung der Darstellungsmittel offenbart sich hier als eine Auseinandersetzung mit Trauer und Tod als zentralen Elementen der Betrachtung. Dies ist insofern wichtig - wenn man die Betrachtungsperspektive erweitert -, als heutzutage das Thema „Tod“ aus dem Leben verdrängt und einer weitestgehenden Tabuisierung unterzogen wird. Frank Feldhusen vertritt sogar den Standpunkt, dass wir gegenwärtig in einer „Trauervermeidungskultur" ${ }^{17}$ leben.

13 In der Darstellung von Moser wird vor allem die Bedeutung einzelner Arien, Rezitative und Chöre hervorgehoben: „Nimmt man aber an innerlichen Höhepunkten die zarte Sopranarie ,Aus Liebe will mein Heiland sterben' (mit Flöte) oder das hochherrliche ,Erbarme dich' für Alt I mit Solovioline, das wie kein zweites unter Bachs stets bedeutenden h-mollStücken die ziervolle Ausdrucksgewalt des Meisters in verklärtestem Lichte zeigt, die vier gewaltigen (meist schmählich gestrichenen) Baßarien oder den phrygischen Choral ,Wenn ich einmal soll scheiden', den jeder evangelische Christ in seiner Sterbestunde hören möchte, das wundersam ruhevolle, von germanischer Naturpoesie erfüllte Arioso ,Am Abend, da es kühle war' oder endlich den schlicht volksliedhaften Begräbnisgesang ,Wir setzen uns mit Tränen nieder', so fühlen wir stets erneut, daß kein Mensch, dem Religion und Musik überhaupt etwas zu sagen haben, ungereinigt und ungetröstet aus einer würdigen Aufführung dieses Werks von dannen gehen kann." Moser, Geschichte der deutschen Musik. Vom Beginn des dreißigjährigen Krieges bis zum Tode Joseph Haydns, 223.

14 Vgl. Wolff, Bachs „Grosse Passion“, 13.

15 Wolff, Bachs „Grosse Passion“, 18.

16 Johann Sebastian Bach, Matthäus-Passion [The Monteverdi Choir, The London Oratory Junior Choir, The English Baroque Soloists, John Eliot Gardiner] (Hamburg: Deutsche Grammophon, 1989), 60.

17 Vgl. Frank Feldhusen, Ich habe deine Tränen gesehen. Aufbauende Aussagen aus Gottes Wort für Zeiten des Abschieds und der Trauer (Norderstedt: Books on Demand, 2012), 29. 
Anders wurde das Ende der irdischen Existenz des Menschen im 18. Jahrhundert wahrgenommen und dargestellt; der Tod galt nicht nur als integraler Teil des Lebens und eine damit untrennbar verbundene und unumgängliche Determinante, er wurde sogar häufig als Ziel des Daseins betrachtet. Eine solche Annahme kann man etwa in den Briefen Mozarts finden, was sich am Beispiel der Korrespondenz mit dem Vater vom 9. Juli 1778 - nur sechs Tage nach dem Tod der Mutter des zu dieser Zeit lediglich 22 Jahre alten Komponisten - zeigen lässt: „durch die gegenwart ihres so leichten und schönen Tods, indemm ich mir vorstelle, wie sie nun in einen augenblick so glücklich wird - wie viell glücklicher das sie nun ist, als wir - so, daß ich mir gewunschen hatte in diesem augenblick mit ihr zu reisen [...]."18 Neun Jahre später schreibt er - mit dem menschlichen Schicksal anscheinend schon ganz versöhnt - im letzten Brief an den Vater: „da der Tod |: genau zu nehmen:| der wahre Endzweck unseres Lebens ist, so habe ich mich seit ein Paar Jahren mit diesem wahren, besten freunde des Menschen so bekannt gemacht, daß sein Bild nicht allein nichts schreckendes mehr für mich hat, sondern recht viel beruhigendes und tröstendes!"19

Zweifelsohne wird auch in der Matthäus-Passion von Bach der Tod nicht tabuisiert, sondern als Übergang zu einem besseren Leben dargestellt. Auch wenn die Manifestationen der Trauer in diesem Werk allgegenwärtig sind, werden sie regelrecht von Inhalten begleitet, die eine erbauende und trostspendende Wirkung haben. Dies lässt sich unter anderem am Motiv des Weinens im Alt-Rezitativ vor der Arie „Buß und Reu knirscht das Sündenherz entzwei“ leicht erkennen: „Du lieber Heiland du, / Wenn deine Jünger töricht streiten, / Daß dieses fromme Weib / Mit Salben deinen Leib / Zum Grabe will bereiten, / So lasse mir inzwischen zu, / Von meiner Augen Tränenflüssen / Ein Wasser auf dein Haupt zu gießen!“20 Tränen, die in der MatthäusPassion gemäß alter Form „Zähren“ genannt werden, erscheinen auch in der darauffolgenden Alt-Arie wobei ein zutiefst religiöser, wenn nicht gar didaktischer Charakter des Textes von Picander zum Vorschein kommt: „Buß und Reu / Knirscht das Sündenherz entzwei, / Daß die Tropfen meiner Zähren / Angenehme Spezerei, / Treuer Jesu, dir gebären."21

Die oben zitierten Textpassagen sind allerdings nicht die einzigen Stellen im majestätischen Werk von J. S. Bach, in dem die Trauer ausdrucksstark dargestellt wird. Explizit veranschaulichtes Weinen der Protagonisten gibt

\footnotetext{
18 Wolfgang Amadeus Mozart, Briefe, hrsg. v. Sarah Donhäuser (Wiesbaden: Marix Verlag, 2006), 107.

19 Mozart, Briefe, 251.

20 Bach, Matthäus-Passion, 64.

21 Bach, Matthäus-Passion, 64.
} 
es auch im Sopran-Rezitativ vor der Arie „Ich will dir mein Herze schenken“: „Wiewohl mein Herz in Tränen schwimmt, / Daß Jesus von mir Abschied nimmt, / So macht mich doch sein Testament erfreut: / Sein Fleisch und Blut, o Kostbarkeit, / Vermacht er mir in meine Hände." ${ }^{22}$

Die zentrale kompositorische Substanz der Matthäus-Passion findet sich allerdings in den madrigalischen Stücken und den lyrischen Betrachtungen der einzelnen Szenen der Leidensgeschichte, wie sie das Libretto von Christian Friedrich Henrici (genannt Picander) darbietet. Vor diesem Hintergrund kann man davon ausgehen, dass die Zusammenarbeit zwischen Bach und dem Libretto-Autor sich auf viele künstlerische Dimensionen erstreckte und höchstwahrscheinlich erfuhr Picanders Text nicht nur Bachs volle Billigung, sondern - eine solche These stellt zumindest Christoph Wolff auf - dieser auch an seiner textlichen Gestaltung Anteil nahm, vor allem soweit es den musikalischen Ablauf betrifft. ${ }^{23}$

In der Matthäus-Passion erscheint eine ausdruckstarke Manifestation der Traurigkeit - abgesehen von den oben genannten und zitierten Beispielen - auch in einer der bekanntesten Alt-Arien der Kirchenmusik überhaupt, nämlich in „Erbarme dich“: „Erbarme dich, / Mein Gott, um meiner Zähren willen! / Schaue hier, / Herz und Auge weint vor dir / Bitterlich. “24 Das Tränen-Motiv wird häufig auch in ausdrucksstarke poetische Phrasen gekleidet, wobei sich nicht selten die Kraftlosigkeit und Verzweiflung der Protagonisten besonders stark offenbaren: „Können Tränen meiner Wangen / Nichts erlangen, / Oh, so nehmt mein Herz hinein! / Aber laßt es bei den Fluten, / Wenn die Wunden milde bluten, / Auch die Opferschale sein!"25

In beiden Passionen von J. S. Bach lässt sich Trauer - auch wenn die dargestellten Emotionen von großer Intensität sind - als Ringen um Ausgewogenheit zwischen verschiedenen Kräften charakterisieren, die nach dem schwerwiegenden Verlust, aber auch in der vorausgreifenden Ahnung eines möglichen privaten Verlustes, auf die Menschen einstürmen. ${ }^{26}$ Dazu kommt

22 Bach, Matthäus-Passion, 70.

23 Vgl. Wolff, Bachs „Grosse Passion“, 13-14.

24 Bach, Matthäus-Passion, 96.

25 Bach, Matthäus-Passion, 108. Für die „große Passion“ ist darüber hinaus symptomatisch, dass in ihrem Anfang gewissermaßen ihr Ende und die weitere Fortsetzung in anderen Werken beschlossen liegt. Christoph Wolff charakterisiert diese Erscheinung mit Berücksichtigung von emotionsbezogenen Aspekte: „Der Eingangschor enthält in sich bereits die dramatische Spannung, die der Schlußchor auflöst - jedoch nur partiell, weil deren eigentliche Auflösung erst mit dem Erklingen der Osterkantate erfolgt.“ Wolff, Bachs „Grosse Passion", 18.

26 Vgl. Hans Goldbrunner, Dialektik der Trauer. Ein Beitrag zur Standortbestimmung der Widersprüche bei Verlusterfahrungen (Berlin: LIT Verlag, 2006), 17. 
der Umstand, dass die Abschiedstrauer, in welcher sich eine besondere Art der negativ gefärbten Emotionalisierung manifestiert, öffentlich und kollektiv ist, sie „besitzt jedoch darüber hinaus eine Dimension, welche die Herzen von vielen Einzelnen in besonderer Weise involviert und dabei heimlich

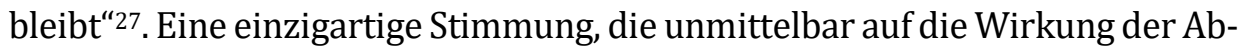
schiedstrauer zurückzuführen ist, lässt sich ebenso im Abschlusschor der Matthäus-Passion in den emotionsbeladenen Worten erkennen: „Wir setzen uns mit Tränen nieder / Und rufen dir im Grabe zu: / Ruhe sanfte, sanfte ruh!“28

\section{„Er hat mich mit Bitterkeit gesättigt“ - Trauerdarstellungen bei Wolfgang Amadeus Mozart}

Wenn man die heutige Todvermeidungskultur in Betracht zieht und sich über die sich über mehrere Jahrhunderte erstreckende kulturelle Evolution der Todeswahrnehmung Gedanken macht, kommt man rasch zu der Überzeugung, dass die immer deutlicher sichtbare mangelnde Bereitschaft, Trauer in der Öffentlichkeit zuzulassen, ebenso zu einem Rückzug Trauernder im öffentlichen Raum geführt hat. Die Manifestation von Gefühlen und Affekten ${ }^{29}$ nach dem Verlust einer Person aus dem nächsten Freundes- oder Verwandtenkreis sowie Trauer selbst, werden folglich immer häufiger zu einer strikt geschützten Privatangelegenheit, „ebenso wie in der modernen Gesellschaft partnerschaftliche und familiäre Intimbeziehungen zu höchst persönlichen „Herzensangelegenheiten “30 erklärt werden.

Anders war es zweifelsohne im 18. Jahrhundert - worauf schon ansatzweise aufmerksam gemacht wurde und was sich wiederum am Beispiel von Mozarts Briefen zeigen lässt - für den das Hinscheiden ein untrennbarer Teil der menschlichen Existenz war. So schreibt er voller Hoffnung und Zuver-

27 Elke Koch, Trauer und Identität (Berlin: Walter de Gruyter, 2006), 272.

28 Bach, Matthäus-Passion, 122.

29 Vor diesem Hintergrund stellt sich die Frage, welche Rolle und Funktion den Affekten in musikalischen Formen zugeschrieben werden kann. Karl Heinrich Wörner und Ekkehard Kreft schlagen folgende Deutung dieser Problematik vor: „Affekte sind emotionale Abstraktionen; der Komponist hat die Aufgabe, solche idealisierte emotionale Zustände im Zuhörer zu erregen. Alle konstituierenden Elemente der musikalischen Komposition wie Tongeschlecht, Tonleiter, Rhythmus, Tempo, Harmonik u. a. können dazu herangezogen werden. So kann der Affekt der Freude durch Dur, schnelles Tempo, vorwiegend konstante und große Intervalle, der Affekt der Trauer durch Moll, langsameres Tempo, häufigen Dissonanzgebrauch u. a. hervorgerufen werden." Karl H. Wörner und Ekkhard Kreft, Geschichte der Musik. Ein Studien- und Nachschlagebuch (Göttingen: Vandenhoeck \& Ruprecht, 1975), 189.

30 Goldbrunner, Dialektik der Trauer, 34. 
sicht am 4. April 1787 an den Vater: „ich lege mich nie zu bette ohne zu bedenken, daß ich vielleicht |: so Jung als ich bin :| den andern Tag nicht mehr seyn werde - und es wird doch kein Mensch von allen die mich kennen sagn können daß ich im Umgange mürrisch oder traurig wäre - und für diese glückseeligkeit danke ich alle Tage meinem Schöpfer und wünsche sie vom Herzen Jedem meiner Mitmenschen. ${ }^{31}$

Eine solche Lebensphilosophie ${ }^{32}$ lässt sich zweifelsohne in zahlreichen musikalischen Werken von Mozart erkennen. Dazu kommt ebenso der Umstand, dass gerade zu seiner Zeit - - und diese Tendenz ist von daher auch für andere Vertreter der Wiener Klassik repräsentativ - sich in den musikalischen Werken ein neues Verständnis der rhetorischen Ebene durchgesetzt hat, das heißt die Aufwertung der Textkenntnis - auch wenn gerade dieser Aspekt schon in früheren Zeiten wichtig und unerlässlich war. Dies kann man unter anderem in Zusammenhang mit der sich im Barock intensiv entwickelnden musikalischen Rhetorik veranschaulichen. Das Voraussetzen der Textkenntnis führte - und dieser Umstand ist bestimmt nicht irrelevant - unmittelbar zu einem Umschwung in der Musikausbildung und zur gesteigerten Pflege philologischer Methoden. ${ }^{33}$

31 Mozart, Briefe, 251. Etwas Ähnliches behauptete Mozart, als er im eigenen Stammbuch nach dem Tod seines Freundes Sigmund Barisani notierte: „Heute, am 2. September dieses nämlichen Jahres, war ich so unglücklich, diesen edlen Mann, liebsten, besten Freund und Erretter meines Lebens, ganz unvermutet durch den Tod zu verlieren. Ihm ist wohl! Aber mir - uns - und allen, die ihn genau kannten - uns wird es niemals wohl werden, bis wir so glücklich sind, ihn in einer besseren Welt wieder auf Nimmerscheiden zu sehen." Carl Bär, Mozart. Krankheit - Tod - Begräbnis (Salzburg: Schriftenreihe der Internationalen Stiftung Mozarteum 1966), 12. Auf eine andere Weise wurden zu dieser Zeit allerdings die Trauerfeiern zelebriert. Carl Bär macht darauf aufmerksam, dass „es unter Regierung Leopolds II., also zur Zeit von Mozarts Tod, überhaupt kein Grabgeleite gab. [...] Nach der Einsegnung der Leiche am Anschluß an die kirchlichen Funktionen war die Trennung zwischen Lebenden und Toten endgültig. Die Bestattung selbst war staatliche Anordnung und vollzog sich unter Ausschluß der Öffentlichkeit." Bär, Mozart. Krankheit - Tod - Begräbnis, 129.

32 Nicht unbedeutend erscheint vor diesem Horizont die Berücksichtigung von philosophischen Strömungen dieser Zeit, zu deren Vertretern David Hume, Adam Smith, Francis Hutcheson, Adam Ferguson und andere Denker gehören, die aus dem kleinen, aber geistig und intellektuell starken Schottland kamen. Sie haben - anders als z. B. die Franzosen - in philosophischen Konzeptionen des Menschen das Prinzip der Gefühle, also breit verstandene irrationale Zustände, befürwortet. Vgl. Sławomir Raube, Nietrwałość, niepewność, smutek. Szkice o literaturze i filozofii kultury (Białystok: Uniwersytet w Białymstoku, 2016), 76.

33 Vgl. Robert Haas, Aufführungspraxis der Musik (Wildpark-Potsdam: Akademische Verlagsgesellschaft Athenaion, 1931), 249-250. Die Bedeutung der musikalischen Rhetorik betont auch Benz: „Dichtung, die so hoch steigt, ist nicht Feindin der Musik - sie ist ihr Kind und ihr Erbe. Gewinnt sie erst musikalische Macht, so wird sie nicht mehr wünschen, daß 
In den vokal-instrumentalen Werken von Mozart, in denen Tränen und Traurigkeit suggestiv, unverborgen und voller innerer Schönheit und poetischer Phrase gezeigt werden, spielen auch zahlreiche Parallelen zu Naturerscheinungen eine gewichtige Rolle. Eine solche Kombination von Inhalten spiegelt sich nicht selten auf der textlichen Ebene und der musikalischen Dimension wider. In der Oper Die Entführung aus dem Serail basiert das Entstehen einer einzigartigen Stimmung von Nachdenklichkeit und Besinnung, die auf das ausdrucksstark dargestellte Schicksal des getrennten Liebespaares zurückzuführen sind, zusätzlich auf zahlreichen bildhaft konzipierten Parallelen. In Konstanzes Arie „Traurigkeit ward mir zum Lose“ kommt diese Tendenz besonders deutlich zum Ausdruck: „Traurigkeit ward mir zum Lose, / Weil ich dir entrissen bin. / Gleich der wurmzernagten Rose, / Gleich dem Gras im Wintermoose, / Welkt mein banges Leben hin. “34 In dieser Arie lässt sich aber gleichzeitig eine Vorahnung und Erwartung bemerken, die aus der Perspektive der Rezipienten Trost spenden und Hoffnung geben kann: Gezeigt werden nämlich Schicksale der Menschen, deren Existenz zwar in affektvollen Umständen platziert wird, nichtsdestotrotz wird dabei der positiven Emotionalisierung eine besondere Rolle zugemessen. Auf der Beispielsebene handelt es sich um Protagonisten, die lieben und geliebt werden und gleichzeitig danach streben, sich mit anderen zu vereinigen, was sie gewissermaßen an die Situation der Hinterbliebenen in ihrer Trauer annähert. 35

Eine andere Stimmung als die oben genannte herrscht zweifelsohne in der Arie von Pamina aus der Oper Die Zauberflöte, wo die Trennung zwi-

\footnotetext{
Musik vergehe, damit das Wort bestehe. Nur Größe duldet das Große ehrfürchtig neben sich." Richard, Benz, Das Ethos der Musik (Offenbach am Main: Verlag Wilh. Gerstung, 1926), 49-50. Mit der musikalischen Rhetorik beschäftigt sich ausführlich auch Nicolaus Harnoncourt: „Jeder Musiker war sich im 17. Jh. und im großen Teil des 18. Jh. darüber bewusst, dass die Kunst, die er betreibt, überdauern soll. Die Rhetorik, mit ihrer ganz komplizierten Terminologie, war nicht nur ein Teil des musikalischen Werks, sondern auch ein obligatorisches Fach in der Schule. Sie gehörte folglich - ähnlich wie Musik - zu der allgemeinen Bildung. Angesichts des Umstands, dass die Affekttheorie von Anfang an einen bedeutenden Einfluss auf die Entwicklung von Barockmusik hatte, war es gleichzeitig auch üblich, sich auf bestimmte Affekte zu sensibilisieren, um sie demnächst an die Hörer weiter leiten zu können. Die Verbindung zwischen Musik und der rhetorischen Kunst war zu dieser Zeit ganz selbstverständlich." Nikolaus Harnoncourt, Muzyka mowq dźwięków. Dialog muzyczny (Warszawa: ME-KOMPS, 2011), 117-118. Wenn nicht anders angegeben, werden alle fremdsprachlichen Texte in der Übersetzung des Autors zitiert.

34 Wolfgang Amadeus Mozart, Die Entführung aus dem Serail, http://www.operafolio.com/libretto.asp?n=Die_Entfuhrung_aus_dem_Serail\&translation=UK.

35 Vgl. S. Brathuhn, Trauer und Selbstwerdung. Eine philosophisch-pädagogische Grundlegung des Phänomens Trauer (Würzburg: Verlag Königshausen \& Neumann, 2006), 46.
} 
schen den Liebenden eine äußerst düstere Stimmung herbeiführt und eindeutig subversive Gedanken aufkommen lässt: „Ach, ich fühl's, es ist verschwunden! / ewig hin der Liebe Glück! - / Nimmer kommt ihr Wonnestunden / meinem Herzen mehr zurück! / Sieh Tamino! diese Tränen / fließen, Trauter, dir allein, / fühlst du nicht der Liebe Sehnen - / so wird Ruh' im Tode sein! - "36 Die gerade zitierte Textpassage aus der in Mozarts Todesjahr 1791 uraufgeführten Oper zeigt deutlich, dass im Durchleben einer Trauer, im bewussten Erleben einer Grenzsituation, der Mensch Einblicke in sich selbst erhält, „die ihm nicht nur bisher ungekannte Einsichten in sein individuelles Selbst, sondern auch Einsichten in sein Menschsein überhaupt gewähren.“37

Eine der signifikantesten Manifestationen der Traurigkeit in Mozarts Werk - abgesehen vom weltberühmten Requiem ${ }^{38}$ - lässt sich allerdings nicht in den Opern, sondern in einer separat geschriebenen Kleinform finden. Gemeint ist die Meistermusik KV 477 in c-Moll, die Mozart für die Freimaurerloge komponierte, in die er am 14. Dezember 1784 aufgenommen wurde. Bei seiner Einführung in den Grad des Meisters, die am 22. April 1785 erfolgte, wurde an die Sage von Hiram erinnert, „der von den Handwerkergesellen getötet wird, die es nicht erwarten können, das Geheimnis der Meisterschaft zu erfahren. “" ${ }^{39}$ Der lateinische Text des Werks drückt - was mit der musikalischen Dimension, insbesondere der meisterhaften Vertonung korrespondiert -eine tiefreichende Bestürzung, Hoffnungslosigkeit und Verzweiflung aus: „Replevit me amaritudinibus, ebriavit me absynthio. Inundaverunt aquae super caput meum: dixi, Perii (Er hat mich mit Bitterkeit gesättigt / und mit Wermut getränkt. / Wasser hat mein Haupt überschwemmt; / da sprach ich: Nun bin ich verloren). ${ }^{40}$

36 Wolfgang Amadeus Mozart, Die Zauberflöte, https://lyricstranslate.com/en/wolfgangamadeus-mozart-die-zauberfl\%C3\%B6te-lyrics.html.

37 Brathuhn, Trauer und Selbstwerdung, 17.

38 Angesichts der Tatsache, dass Mozarts Requiem zweifelsohne zu den bekanntesten Beispielen der musikalisch ausgedrückten Trauer in der Musikgeschichte gehört und in unzähligen Abhandlungen äußerst genau und vieldimensional analysiert wurde, wurde dieses Meisterwerk im Rahmen des vorliegenden Beitrags nicht separat dargestellt und bearbeitet. Erwähnenswert sind nichtsdestotrotz einige repräsentative Fachliteraturbeispiele: Matthias Korten, Mozarts Requiem und seine Bearbeitungen; Beatrice Ebel Die Salzburger Requiemtradition im 18. Jahrhundert. Untersuchungen zu den Voraussetzungen von Mozarts Requiem; Christoph Wolff Mozarts Requiem. Geschichte, Musik, Dokumente, Partitur des Fragments; Heinz Gaertner Mozarts Requiem und die Geschäfte der Constanze M.; Heinz-Alfred Seelbach, Das Wort-Ton-Verhältnis in den Requiem-Vertonungen von Mozart und Verdi.

39 Jean-Yves Bras, „Meistermusik KV 477“, übers. v. Heidi Fritz, in Wolfgang Amadeus Mozart, Messe en ut mineur [La Chapelle Royale, Collegium Vocale, Orchestre des Champs Élysées, Philippe Herreweghe] (Arles: harmonia mundi, 1992), 19.

40 Mozart, Messe en ut mineur, 23. 


\section{„Leichte Segler in den Höhen“ - Melancholie und Trauermotive in der Musik Ludwig van Beethovens}

Die musikalisch dargestellten Tränen- und Traurigkeitsmotive erscheinen auch in den Liedern des in Bonn geborenen Komponisten, auch wenn diese, rein chronologisch gesehen, nicht mehr zum 18., sondern schon zum Anfang des 19. Jahrhunderts gehören. Als Beispiel kann das Lied An die Hoffnung zu den Worten von Friedrich Hölderlin dienen: „Die du so gern in heil'gen Nächten feierst / Und sanft und weich den Gram verschleierst, / Der eine zarte Seele quält, / O Hoffnung! Laß, durch dich empor gehoben, / Den Dulder ahnen, daß dort oben / Ein Engel seine Tränen zählt!“41

Schon die erste Analyse des gerade zitierten Liedtextes führt zu der Feststellung, dass Traurigkeit hier als jene Art der Selbsterfahrung und Selbstfindung erscheint, bei der zusätzlich der private Charakter dieses Gefühls in den Vordergrund rückt. Vor allem manifestieren sich in diesem Zusammenhang die mit Traurigkeit eng verzahnten Emotionen in rückblickenden Nachgedanken, wenn bereits - etwas zugespitzt formuliert - eine lange Strecke des beschwerlichen Trauerweges zurückgelegt ist. Erst dann wird nämlich „die persönliche Bereicherung greifbar, die der Trauer gleichzeitig eine kritisch-emanzipatorische Haltung gegenüber oberflächlichen Leitbildern der sozialen Umwelt verleiht." ${ }^{42}$

Manifestationen der Traurigkeit gibt es in Beethovens Werk auch im Lied Leichte Segler in den Höhen (der Text stammt von Alois Isidor Jeitteles), wo ausgerechnet dieses Gefühl und das Tränen-Motiv eine besonders stark emotionalisierende Funktion haben: „Flüstr' ihr zu mein Liebesflehen, / Laß sie, Bächlein klein und schmal, / Treu in deinen Wogen sehen / Meine Tränen ohne Zahl!“43 Der Stellenwert der Trauer hat hier eine zusätzliche Dimension: Er steht einerseits für Identitätskonstruktionen, die sich auf der Textebene offenbaren, ist dennoch andererseits nicht allein durch die Selbstthematisierung des Subjekts im Klagemonolog begründet. Maßgeblich ist vielmehr, dass auf der Ebene der musikalischen Darstellung - wenn nicht gar Inszenierung - neben Reaktionen und Interaktionen zwischen den Figuren auch differenzierte Strategien zu konstatieren sind, mittels derer komplexe Inhalte, Gefühle und Identitäten entworfen werden. ${ }^{4}{ }^{4}$

41 Friedrich Hölderlin, An die Hoffnung, https://www.lieder.net/lieder/get_text.html?TextId $=15806$.

42 Hans Goldbrunner, Dialektik der Trauer. Ein Beitrag zur Standortbestimmung der Widersprüche bei Verlusterfahrungen (Berlin: LIT Verlag, 2006), 35.

43 Alois Jeitteles. Leichte Segler in den Höhen, https://www.lieder.net/lieder/assemble_ texts.html?SongCycleId=128.

44 Vgl. Elke Koch, Trauer und Identität (Berlin: Walter de Gruyter, 2006), 284-285. Eine starke Affektbildung, wie jene in den oben zitierten Liedern von Ludwig van Beethoven, lässt sich 


\section{Die sieben letzten Worte unseres Erlösers am Kreuze - stimmungsvolle Trauerdarstellungen bei Joseph Haydn}

Aus der musikhistorischen Perspektive gesehen, gibt es wohl kaum eine interessantere Epoche als jene der sogenannten Klassik, zu der nicht nur zwei der bereits erwähnten Komponisten (Mozart und Beethoven) gehören, sondern auch der in Rohrau geborene spätere Kapellmeister am Hof der adligen Familie Esterházy, Joseph Haydn. Wegen ihrer geografischen Wirkungsstätte wird diese Epoche in der Musik nicht zu Unrecht mit dem Zusatz „Wiener Klassik“ versehen. Angesichts der auffälligen Unterschiede in der Bewertung des CEuvres der oben genannten Komponisten wird Joseph Haydn im Unterschied zu seinen beiden Großmeisterkollegen auch heute immer noch weniger beachtet - diesen Standpunkt vertritt zumindest Frank Huss. Und dies sowohl als Mensch, als auch als Komponist. Als Beleg dafür kann der Umstand herangezogen werden, dass er lange Zeit der Unbekannteste der drei großen Meister war und sein komplett aufgearbeitetes Gesamtwerk erst seit etwa vierzig Jahren vorliegt. ${ }^{45}$

Bedeutend im Zusammenhang mit musikalisch dargestellten Emotionen und Traurigkeitsmanifestationen in den Werken von Haydn erscheint erstens das Oratorium Die Schöpfung, das am 19. März 1799, dem Tag des heiligen Josef, dem breiteren Publikum präsentiert wurde, zweitens Die sieben letzten Worte unseres Erlösers am Kreuze, das zwei Tage zuvor im Zuge einer Benefizveranstaltung aufgeführt wurde. ${ }^{46}$ Gerade das zweite Werk erscheint als besonders erwähnenswert, denn hier kommt eine einzigartige Verbindung von biblischen Texten und einer poetischen Bearbeitung und Erweiterung zum Vorschein. Dies lässt sich etwa am Beispiel folgender Worte veranschaulichen: „Frau, hier siehe deinen Sohn, / Und du, siehe deine Mutter! / Mutter Jesu, die du trostlos / Weinend, seufzend bei dem Kreuze standst. / Und die Qualen seines Leidens / In der Stund' des bittern Scheidens, / Siebenfach in dir empfandst." 47

Das Weinen fungiert sowohl in der musikalisch verlaufenden Darstellung vor dem Publikum als auch in einer nicht-öffentlichen Situation und

ebenso in vokalen Kurzformen „Sehnsucht“, „Des Einsamen Klage“, „Der Schmetterling auf einem Grabmal“ oder „Trost in Tränen“ des heute fast völlig unbekannten deutschen Komponisten Johann Friedrich Reichardt (1752-1814) finden.

45 Vgl. Frank Huss, Joseph Haydn. Das unterschätzte Genie (Wien: Hollitzer Wissenschaftsverlag, 2013), 8.

46 Vgl. Huss, Joseph Haydn. Das unterschätzte Genie, 172.

47 Joseph Haydn, Die sieben letzten Worte [Chamber Choir of Europe, Kurpfälzisches Kammerorchester Mannheim, Nicol Matt, Brilliant Classics] (Mannheim: Kurpfälzisches Kammerorchester, 2002), 5 . 
Form - was sich ebenso am Beispiel des Oratoriums von Haydn zeigen lässt - als Element ritualisierter Kommunikation. Es besitzt des Weiteren auch jene weitere Funktion, die den sozialen und kollektiven Aspekt verdeutlicht. Gemeint ist hier vor allem der Ausdruck einer Treuebindung; folglich erscheinen die musikalisch dargestellten Tränen einerseits als sozial modellierte Geste in angemessenen öffentlichen Situationen, andererseits als unmittelbarer Verzweiflungs- und Furchtausdruck.48

Für Haydns Oratorien ist darüber hinaus der Umstand relevant, dass die Qualität seiner Vokalwerke unmittelbar von Inhalt und Art des Textes abhängig ist. Vor diesem Horizont muss man darauf verweisen, dass es zur Zeit der Wiener Klassik eher unüblich war, den Wert eines Vokalwerkes als vom Text unabhängig begreifen zu wollen. Übrigens ist eine solche Tendenz bis heute erhalten geblieben, schließlich - und dieser Gedanke knüpft an die hegelianische Tradition an - darf keinem Künstler der Stoff, den er behandelt, gleichgültig bleiben und dem Musiker umso weniger. Denn ausgerechnet dank der Textkenntnis kann er die nähere epische, lyrische und dramatische Form des Inhalts schon im Voraus bearbeiten und feststellen. ${ }^{49}$

Wenn man die oben dargestellten Argumente einer näheren Analyse unterzieht, kommt man auch zu der Überzeugung, dass die musikalische Stimulierung einen spezifischen Einfluss auf die menschliche Rezeption hat. Sie bildet zwar keine außerordentlich starke Signalquelle, insbesondere wenn sie ohne weitere affektive Stimulierung (z. B. mittels eines Bildes) vorkommt, sie ist dennoch imstande, die Stimmung des Empfängers bedeutend zu beeinflussen. Die Wahrnehmung der Musik ist unabhängig davon eine der populärsten Methoden, die eigene Stimmung entsprechend zu regulieren. Ihre Wirkung auf unsere allgemeine geistige Kondition kann entweder durch den physiologischen oder psychischen Mechanismus erfolgen. Im ersten Fall spielt vor allem das Tempo des musikalischen Werks eine große Rolle, das sich folglich unmittelbar auf die affektive Erregung auswirkt, wodurch auch die Stimmung reguliert wird. Der zweite Mechanismus basiert grundsätzlich auf der Assoziation der gehörten Musik mit früheren Ereignissen und Objekten, die einer positiven oder negativen Bewertung unterzogen werden. ${ }^{50}$

48 Vgl. Koch, Trauer und Identität, 273.

49 Vgl. Georg Wilhelm Friedrich Hegel, Vorlesungen über die Ästhetik, zit. nach: Hanns Eisler, Materialien zu einer Dialektik der Musik (Leipzig: Verlag Philipp Reclam, 1976), 241.

50 Vgl. Andrzej Rynkiewicz, Smutek. Analiza psychologiczna (Warszawa: Wydawnictwo Naukowe Scholar, 2014), 129. 


\section{Schlussfolgerungen}

Die oben dargestellte Manifestation von Emotionen in den Werken von J. S. Bach und Vertretern der Wiener Klassik untermauert die These, dass im Falle der Darstellungen von Trauer und Traurigkeit sowohl der musikalischen als auch der rhetorischen Ebene eine besondere Rolle beigemessen werden muss. Schon vor nahezu hundert Jahren machte Richard Benz darauf aufmerksam, dass man aber insbesondere der Interpretation der musikalischen Inhalte mehr Beachtung schenken muss, weil wir häufig nicht darauf achten, „welche Musik den Menschen umtönt, den werdenden wie den erwachsenen - wir wissen nicht, oder wollen nicht wissen, daß der Kunst eine sittliche Macht innewohnt; daß diese Macht gewußt werden muß, um die entsittlichende zu erkennen und von ihr zu scheiden. ${ }^{\text {"51 }}$ Andererseits ist in diesem Zusammenhang der Umstand von Belang, dass die Schöpfer-Macht der Musik an das Wort übergeht und folglich auch ihr gewordenes Sein, ihre Wirkung und ihr Erlebnis zum Mysterium ${ }^{52}$ der Menschheit werden. In einer solchen Auffassung erscheint ferner Musik, so Benz, als „ewiger Schöpfung Urquell“, denn sie gestaltet nicht nur Künste allein, sondern auch das Leben selbst. Sie ist auch imstande, einen neuen Menschen zu kreieren und zwar ein Individuum, das nicht mehr zerrissen ist und in der dualen Abgrenzung zwischen Verstand und Sinnlichkeit existiert bzw. in bloßen Begriffen den Geist und Sinnenfreude zu besitzen strebt. Vielmehr entsteht durch die Wirkung der Musik ein Typ des Menschen, so Benz, „in dem Geist und Sinne geeint sind: der den Geist im Geheimnis der Kunst erfuhr, und nun auch in sich selbst das Geheimnis wiederfindet; im Geheimnis und aus dem Geheimnis zutiefst zu innerlichst lebt." ${ }^{3}$

\section{References}

Bach, Johann Sebastian. Johannes-Passion [The Monteverdi Choir, The English Baroque Soloists, John Eliot Gardiner]. Hamburg: Polydor International, 1986.

51 Benz, Das Ethos der Musik, 35.

52 Auf ähnliche Weise kann man die Wahrnehmung des musikalischen Ethos mit dem Phänomen des künstlerischen Mysteriums verbinden: „Das Ethos der Musik erfahren, heißt, das Mysterium des Geistes verstehen, das sich der neueren Welt nur im musikalischen Act offenbart hat. Es heißt nicht, dieses Geheimnis mit dem Verstande durchdringen wollen; es wird (mit Begriffen unsagbar) nur im Erlebnis der Sinne erfahren." Benz, Das Ethos der Musik, 42.

53 Benz, Das Ethos der Musik, 50. 
Bach, Johann Sebastian. Matthäus-Passion [The Monteverdi Choir, The London Oratory Junior Choir, The English Baroque Soloists, John Eliot Gardiner]. Hamburg: Deutsche Grammophon, 1989.

Bär, Carl. Mozart. Krankheit - Tod - Begräbnis. Salzburg: Schriftenreihe der Internationalen Stiftung Mozarteum, 1966.

Benz, Richard. Das Ethos der Musik. Offenbach am Main: Verlag Wilh. Gerstung, 1926.

Bras, Jean-Yves. "Meistermusik KV 477." Translated by Heidi Fritz. In Mozart, Wolfgang Amadeus. Messe en ut mineur [La Chapelle Royale, Collegium Vocale, Orchestre des Champs Élysées, Philippe Herreweghe], Arles: harmonia mundi, 1992.

Brathuhn, Sylvia. Trauer und Selbstwerdung. Eine philosophisch-pädagogische Grundlegung des Phänomens Trauer. Würzburg: Verlag Königshausen \& Neumann, 2006.

Feldhusen, Frank. Ich habe deine Tränen gesehen: Aufbauende Aussagen aus Gottes Wort für Zeiten des Abschieds und der Trauer. Norderstedt: Books on Demand, 2012.

Fischer von, Kurt. "Historische und frömmigkeitsgeschichtliche Traditionen der Passion." In Bach, Jochann Sebastian. Johannes-Passion [The Monteverdi Choir, The English Baroque Soloists, John Eliot Gardiner]. Hamburg: Polydor International, 1986.

Goldbrunner, Hans. Dialektik der Trauer. Ein Beitrag zur Standortbestimmung der Widersprüche bei Verlusterfahrungen. Berlin: LIT Verlag, 2006.

Haas, Robert. Aufführungspraxis der Musik. Wildpark-Potsdam: Akademische Verlagsgesellschaft Athenaion, 1931.

Harnoncourt, Nikolaus. Muzyka mowq dźwięków. Dialog muzyczny. Translated by Magdalena Czajka. Warszawa: ME-KOMPS, 2011.

Haydn, Joseph. Die sieben letzten Worte [Chamber Choir of Europe, Kurpfälzisches Kammerorchester Mannheim, Nicol Matt, Brilliant Classics]. Mannheim: Kurpfälzisches Kammerorchester, 2002.

Eisler, Hanns. Materialien zu einer Dialektik der Musik. Leipzig: Verlag Philipp Reclam, 1976.

Huss, Frank. Joseph Haydn. Das unterschätzte Genie. Wien: Hollitzer Wissenschaftsverlag, 2013.

Hölderlin, Friedrich. An die Hoffnung. https://www.lieder.net/lieder/ get_text.html?TextId=15806.

Jeitteles, Alois. Leichte Segler in den Höhen. https://www.lieder.net/lieder/ assemble_texts.html? SongCycleId=128.

Jöde, Fritz. Musik und Erziehung. Ein pädagogischer Versuch und eine Reihe Lebensbilder aus der Schule. Berlin: Georg Kallmeyer Verlag, 1932. 
Joyner, Elisabeth. Erbarme Dich. The Temptation of Peter in Bach's St. Matthew Passion. https://earthandaltarmag.com/posts/erbarmedichthe-temptation-of-peter-in-bachs-st-matthew-passion.

Koch, Elke. Trauer und Identität. Berlin: Walter de Gruyter, 2006.

Moser, Hans Joachim. Geschichte der deutschen Musik. Vom Beginn des dreißigjährigen Krieges bis zum Tode Joseph Haydns. Stuttgart und Berlin: J. S. Cotta'sche Buchhandlung Nachfolger, 1923.

Mozart, Wolfgang Amadeus. Briefe, edited by Sarah Donhäuser. Wiesbaden: Marix Verlag, 2006.

Mozart, Wolfgang Amadeus. Die Entführung aus dem Serail. http://www. operafolio.com/libretto.asp?n=Die_Entfuhrung_aus_dem_Serail\&translation=UK.

Mozart, Wolfgang Amadeus. Die Zauberflöte. https://lyricstranslate.com/ en/wolfgang-amadeus-mozart-die-zauberfl\%C3\%B6te-lyrics.html.

Pastor, Willy. Die Geburt der Musik. Eine Kulturstudie. Leipzig: Fritz Eckardt Verlag, 1910.

Raube, Sławomir. Nietrwałość, niepewność, smutek. Szkice o literaturze i filozofii kultury. Białystok: Uniwersytet w Białymstoku, 2016.

Rynkiewicz, Andrzej. Smutek. Analiza psychologiczna. Warszawa: Wydawnictwo Naukowe Scholar, 2014.

Schaeben, Ulrike. Trauer im humanistischen Dialog. München/Leipzig: Saur Verlag, 2002.

Wolff, Christoph. “Bachs 'Grosse Passion'.” In Bach, Johann Sebastian. Matthäus-Passion [The Monteverdi Choir, The London Oratory Junior Choir, The English Baroque Soloists, John Eliot Gardiner]. Hamburg: Deutsche Grammophon, 1989.

Wörner, Karl H., and Ekkhard Kreft. Geschichte der Musik: ein Studien- und Nachschlagebuch. Göttingen: Vandenhoeck \& Ruprecht, 1975.

\section{„Können Tränen meiner Wangen nichts erlangen?“ Manifestationen der Traurigkeit in der deutschen Musik des 18. Jh.}

\footnotetext{
Abstract: Emotionen und Gefühle wie Traurigkeit, Enttäuschung oder Verzweiflung sind nicht nur für die breit verstandene deutschsprachige Literatur signifikant. Ihren Manifestationen kann man auch in den Werken der vokal-instrumentalen Musik begegnen, deren Blütezeit in das 18. Jh. fällt, insbesondere in die Zeit zwischen dem Entstehen von Bachs JohannesPassion BWV 245 (1724) und der Erstaufführung von majestätischen Oratorien wie Die Schöpfung und Die sieben letzten Worte unseres Erlösers am Kreuze von Joseph Haydn (179698). Das Ziel des vorliegenden Beitrags ist das emotionale Potenzial ausgewählter Texte vor dem Hintergrund der musikalischen Rhetorik zu zeigen, sowie die Werke von berühmten
} 
deutschen Komponisten des $18 \mathrm{Jh}$. in einen breiteren kulturellen und literarischen Kontext einzubinden.

Schlüsselwörter: Passionsmusik von Johann Sebastian Bach, Wiener Klassiker, Emotionen und Affekte.

\title{
„Czy łzy moje niczego nie zmienią?" Manifestacje smutku w muzyce niemieckiej XVIII wieku
}

\begin{abstract}
Abstrakt: Emocje i uczucia, jak smutek, rozczarowanie czy zwątpienie, nie są jedynie cechą charakterystyczną narracji obecnych w szeroko rozumianej literaturze niemieckojęzycznej. Manifestacje różnych stanów emocjonalnych napotkać można w licznych dziełach wokalnoinstrumentalnych, których okres świetności przypada na wiek XVIII, w szczególności na dekady zamykające się w ramach czasowych, wyznaczonych przez Pasję wg św. Jana Johanna Sebastiana Bacha (BWV 245) z roku 1724 oraz majestatyczne oratoria Stworzenie Świata i Siedem Ostatnich Słów Chrystusa na Krzyżu Josepha Haydna (1796-98). Celem niniejszego artykułu jest pokazanie potencjału afektywnego wybranych tekstów będących w orbicie oddziaływania zjawiska retoryki muzycznej oraz naświetlenie dzieł sławnych niemieckich kompozytorów osiemnastego stulecia, umieszczonych w szerszym kontekście kulturowym i literackim.
\end{abstract}

Słowa kluczowe: muzyka pasyjna Johanna Sebastiana Bacha, klasycy wiedeńscy, emocje i afekty. 